\title{
Achados ecocardiográficos em pacientes com fibrilação atrial na monitorização
}

\section{eletrocardiográfica de $24 \mathrm{~h}$}

\author{
Echocardiographic findings in patients with atrial fibrillation evidenced in electrocardiographic \\ monitorization
}

Hallazgos ecocardiográficos en pacientes con fibrilación atrial evidenciada en monitorización electrocardiográfica

\section{Resumo}

Fibrilação atrial (FA), patologia de disfunção elétrica cardíaca de alta morbimortalidade que possui 2-3\% de prevalência global e causa aumento em duas vezes de mortalidade geral, detectado no Eletrocardiograma. O exame de ecocardiografia transtorácico (ETT) é um método de imagem da clínica cardiológica para avaliação morfológica, funcional e hemodinâmica. Este estudo teve o objetivo de determinar achados nos pacientes com FA pela análise de parâmetros do exame Holter e ETT, apresentar o padrão de distribuição de dimensões cardíacas nos pacientes com FA e correlacionar a presença de outras alterações descritivas que não FA nos pacientes com e sem FA. Foi observado o holter de 3988 pacientes, naqueles com FA foi realizado ETT e analisados os achados morfológicos destes exames. Os resultados foram apresentados em estimativas pontuais e medidas de dispersão foram calculadas por intervalos de confiança a $95 \%$. Considerou-se o valor de p bicaudal < 0,05 como significância estatística. Dentre os 3988 pacientes analisados, 3730 apresentaram ritmo sinusal e 258 FA. Observou-se um índice de volume de átrio esquerdo (IVAE) médio de $38,82( \pm 17,95) \mathrm{ml} / \mathrm{m} 2$ nos pacientes com FA. Além disso, diâmetro do átrio esquerdo possuía percentil 75 médio de $44 \mathrm{~mm}$ nos pacientes com FA. Ainda, os pacientes tiveram prevalência de alterações mínimas, moderadas e graves de 53,94\%, 10,63\% e 5,51\%, respectivamente. Observou-se maior diferença significativa $(<0,001)$ no IVAE. Comparando ao ritmo sinusal, indivíduos com FA apresentaram maior IVAE $(<0,001)$ e maior presença de alterações descritivas consideradas moderadas ou graves $(<0,001)$, que não FA.

Palavras-chave: Fibrilação atrial; Eletrocardiografia ambulatorial; Ecocardiografia.

\begin{abstract}
Atrial fibrillation (AF), a pathology characterized as heart's electrical dysfunction with high morbidity and mortality, has a $2-3 \%$ global prevalence and causes two-fold increase in mortality. The transthoracic echocardiography exam (TTE) is an imaging method used in cardiology clinic with the aim of a morphological, functional and hemodynamic evaluation. This study had the objective to determine findings in patients with AF by analyzing the holter and TTE's parameters, present the distribution pattern of the cardiac dimensions in these patients and correlate the presence of other descriptive alterations, which are not AF, between those in sinusal rhythm and AF. The holter of 3988 individuals were observed, those which had AF performed an TTE and their morphological findings were. The results were presented in punctual estimates and the dispersion measures were calculated by trust intervals of $95 \%$. The twotailed $p$ value of $<0,05$ was considered of statistical significance. Among the 3988 patients analyzed, 3730 patients were in sinus rhythm and 258 individuals demonstrated AF. The average left atrial volume index (LAVI) found was $38,82( \pm 17,95) \mathrm{ml} / \mathrm{m} 2$ in those with AF. Furthermore, the left atrium diameter had a more significant 75 percentile of $44 \mathrm{~mm}$. Additionally, these patients had a prevalence of minimal, moderate and severe alterations of 53,94\%, 10,63\% and $5,51 \%$, respectively. The finding with largest significant difference $(<0.001)$ was the LAVI. In comparison to those in sinusal rhythm, AF patients showed a LAVI $(<0,001)$ and a larger presence of other descriptive alterations considered moderate or severe $(<0,001)$, that weren't AF.
\end{abstract}

Keywords: Atrial fibrillation; Electrocardiography, ambulatory; Echocardiography. 


\begin{abstract}
Resumen
Fibrilación atrial (FA) és una patología caracterizada por disfunción eléctrica cardíaca con alta morbilidad y mortalidad, tiene prevalencia global del 2-3\%. El examen de ecocardiografía transtorácica (ETT) es un método de imagen utilizado en la clínica de cardiología. Este estudio tuvo como objetivo determinar hallazgos en pacientes con FA mediante el análisis de los parámetros de holter y ETT, presentar el patrón de distribución de las dimensiones cardíacas y correlacionar la presencia de otras alteraciones descriptivas, que no sea FA, entre los que están en ritmo sinusal y AF. Se observó el holter de 3988 individuos, a los que tenían FA se les realizó un ETT y sus hallazgos morfológicos fueron analizados. Los resultados se presentaron en estimaciones puntuales y las medidas de dispersión se calcularon mediante intervalos de confianza del 95\%. El valor de p de <0,05 se consideró de significación estadística. Entre los 3988 pacientes analizados, 3730 pacientes estaban en ritmo sinusal y 258 individuos demostraron FA y en estos el índice de volumen atrial izquierdo (IVAI) promedio encontrado fue de 38,82 ( $\pm 17,95)$ $\mathrm{ml} / \mathrm{m} 2$. Además, el diámetro del átrio izquierdo tenía un percentil $75 \mathrm{de} 44 \mathrm{~mm}$. Además, estos pacientes tuvieron una prevalencia de alteraciones mínimas, moderadas y severas de 53,94\%,10,63\% y 5,51\%, respectivamente. El hallazgo con mayor diferencia significativa $(<0,001)$ fue el IVAI. En comparación con los que estaban en ritmo sinusal, los pacientes con FA mostraron un IVAI mayor $(<0,001)$ y una mayor presencia de alteraciones descriptivas $(<0,001)$, que no FA.
\end{abstract}

Palabras clave: Fibrilación atrial; Electrocardiografía ambulatoria; Ecocardiografía.

\title{
1. Introdução
}

Use o parágrafo como modelo $\mathrm{O}$ sistema cardiovascular é vinculado ao aumento de enfermidades relacionadas à senescência (Soeki, et al., 2018). Uma dessas é a Fibrilação Atrial (FA), patologia caracterizada em disfunção elétrica do coração com taquiarritmia supraventricular e hipocontratilidade atrial (Jonas, et al., 2018). A FA possui 2-3\% de prevalência global e espera-se um aumento em 2,3 vezes devido a senescência da população e às comorbidades associadas a estes pacientes (Hindricks, et al., 2021). Portando, a FA é responsabilizada pelo aumento da morbidade e mortalidade em duas vezes nos portadores, a partir de maior risco para eventos tromboembólicos, disfunção cardíaca e demência (Jonas, et al., 2018).

Ainda, mesmo com sua importância clínica e prognóstica, a FA pode ser subdiagnosticada, principalmente nos oligossintomáticos e pela transitoriedade dos episódios, até que ocorram eventos tromboembólicos e cardiovasculares de maior gravidade e alta morbimortalidade, sendo o Acidente Vascular Encefálico (AVE) o mais grave (Field, et al., 2019). Destes, um terço são criptogênicos com $30 \%$ associados a FA oculta (Ricci, 2018). O rastreamento da FA, é realizado pelo eletrocardiograma (ECG), com pelo menos 30 segundos do padrão típico da FA: ausência de onda P e intervalos RR irregulares, principalmente em pacientes com FA Observável ou com manifestações clínicas típicas. Naqueles com FA subclínica e/ou crônica, pode se fazer necessária a monitorização eletrocardiográfica ambulatorial de 24h ou, em alguns casos, de até 72h (Kirchhof, et al., 2017).

Durante a avaliação achados ecocardiográficos são associados a FA: átrio esquerdo (AE) aumentado, disfunção diastólica, frequência cardíaca baixa, intervalo RR máximo longo, pausas sinusais e Contrações Atriais Prematuras (CAP) são associadas ao desenvolvimento de FA através de impacto ao miocárdio do AE causando disfunção mecânica e remodelação (Soeki, et al., 2018; John, Hirsch \& Stoddard 2018). Ainda, o aumento do AE, avaliado através do índex de volume e diâmetro do AE, funciona como preditor do desenvolvimento da FA (Ricci, et al., 2018). Além disso, pode orientar o acompanhamento dos portadores, sendo 4,2 cm considerada a medida a partir da qual orienta-se um seguimento mais intenso e manejo otimizado do estado clínico desses pacientes (Zegkos, et al., 2017).

Portanto, a avaliação clínica, imaginológica e eletrofisiológica, pode orientar do rastreamento ao manejo e otimização das decisões clínicas para melhor prognóstico. Por fim, conhecimento sobre os achados encontrados nesses exames, em especial o ecocardiograma, leva a melhor acompanhamento para o portador da FA.

\section{Metodologia}

Trata-se de um estudo analítico, observacional, transversal e descritivo (Estrela, 2018), com coleta de dados em 
modelo "all comers", realizado em forma de registro. A população da amostra foi arrolada a partir do atendimento ambulatorial realizado na instituição de referência cardiológica com certificado de acreditação Qualis 3. Além disso, o levantamento de dados e a análise do Holter de 24 horas foram realizados nesta instituição.

Os indivíduos foram informados dos objetivos da pesquisa, tendo sido adicionados aos bancos de dados os que consentiram em participar da pesquisa e preencheram o termo de consentimento livre e esclarecido. A coleta de dados se deu de maneira prospectiva e consecutiva, entre dezembro de 2011 e agosto de 2020.

Foram selecionados 3.988 pacientes dos quais realizou-se um estudo ancilar com uma amostra de 258 pacientes portadores de fibrilação atrial e que realizaram ecocardiograma transtorácico, entre adultos e idosos ativos de ambos os gêneros, em satisfatório estado cognitivo e em plena capacidade funcional. Os pacientes classificados como portadores de fibrilação atrial apresentaram episódios sustentados, ou seja, de longa duração, contínuo, ou seja, durante todo o registro de Holter, e frequentes paroxismos não sustentados, ou seja, sucessivos episódios com duração inferior a 120 segundos.

Ainda, as variáveis observadas em 258 pacientes foram diâmetro da aorta em milímetros, diâmetro do átrio esquerdo, diâmetro diastólico do ventrículo esquerdo, fração de ejeção, diâmetro septal. Além destes, somente 257 observaram diâmetro sistólico do ventrículo esquerdo, em 254 avaliou-se os achados eletrocardiográficos nos quais, 244 observaram a função diastólica, em 101 pacientes foi analisado o volume do átrio esquerdo indexado. Ainda, em um número reduzido de pacientes foram observadas variáveis adicionais tais como: 72 obtiveram a razão E:E linha, em 65 observou-se a onda E linha, em 64 a onda E, e em somente 6 viu-se a onda A e a razão E:A.

O motivo mais comum para a realização do exame foi avaliação cardiológica de rotina em assintomáticos ou investigação de sinais e sintomas inespecíficos, como palpitações, tonturas ou precordialgia atípica.

Foram incluídos na pesquisa pacientes que possuíam todos os critérios seguintes: aceitaram participar da pesquisa, que possuíssem uma idade igual ou superior a quarenta anos, que tivessem exame realizado em situação ambulatorial, com capacidade de deambular sem auxílio e de compreender as indagações do formulário sociodemográfico.

Ademais, foram excluídos da pesquisa pacientes que possuíssem pelo menos um dos seguintes critérios: portadores de diabetes mellitus insulinodependente; usuários de marcapasso; histórico pessoal de cirurgia cardíaca; pacientes em uso de simpaticomiméticos, antagonistas dos canais de cálcio (verapamil e diltiazem), digitálicos, drogas antiarrítmicas, betabloqueadores ou fármacos com ação direta na FC (a exemplo do nebivolol); pacientes com frequência cardíaca média $<60$ bpm ou > 90 bpm; pacientes com alterações transitórias de segmento ST e ondas T no registro de Holter; traçado de qualidade insatisfatória ou incidência de mais de 5\% de artefatos e/ou gravação no Holter com duração inferior a 22 horas.

Foi solicitado dos participantes dados sociodemográficos (idade, cor autorreferida, estado civil, nível de escolaridade) e informações sobre sintomas (palpitação, tonturas, precordialgia), hábitos de vida (tabagismo, consumo de álcool, atividade física), além de responder a perguntas indicadoras do estado geral (uso de medicamentos, e internações hospitalares ou admissões na urgência nos últimos doze meses).

O formulário preenchido integralmente durante a entrevista. As perguntas, aplicadas sempre na mesma ordem, são em maioria com opções de resposta predeterminadas. Eventuais dúvidas em relação ao seu conteúdo foram esclarecidas até o ponto em que se constatou que o paciente compreendeu o suficiente para respondê-las. Devido a população com parcela de idosos suscetíveis a algum tipo de dificuldade visual, foi evitado questionário autoaplicável, preferindo-se formulário preenchido pelo entrevistador, com o intuito de garantir o entendimento das questões durante a conversa.

O analisador de Holter foi do tipo "digital", modelo CardioSmart Professional CS 540. O gravador, um CardioLight da Cardios, também digital, portador de "Memory Card" e dotado de cabo de quatro vias, apto a produzir traçados de 3 canais, sendo o primeiro V1 modificado, o segundo D2 modificado e o terceiro V5 modificado. Dentre os vários sistemas de Eletrocardiografia Ambulatorial, o modelo digital de gravação em três canais simultâneos foi rigorosamente validado por 
diversos pesquisadores (DiMarco \& Philbrick, 1990; Kennedy, 2006; Morganroth, 1985). Emblemáticas associações cardiológicas internacionais - American College of Cardiology e American Heart Association (Crawford, et al., 1999) consubstanciaram a metodologia. Os passos referentes ao procedimento são: instalação; gravação; transferência dos dados; processamento automático; análise médica, elaboração de laudo e emissão de relatório.

Durante a instalação, ocorre a limpeza da pele do tórax, a aplicação dos eletrodos, a preparação do "Memory Card" e a conexão do cabo ao gravador digital. Todo esse processo é realizado por funcionário devidamente treinado, visando garantir a obtenção de sinal eletrocardiográfico de qualidade. O paciente foi orientado a exercer suas atividades normais por 24 horas, devendo evitar contato de líquidos com o aparelho e os eletrodos. Tanto as atividades diurnas quanto o período noturno, incluindo o sono, são incorporadas à análise.

A duração de cada registro de Holter variou entre 22 horas e 24 horas. A transferência de dados é feita assim que o paciente retornava à clínica, sendo imediatamente realizado o processamento automático. A análise do exame e a produção do laudo final esteve a cargo do mesmo médico, cardiologista experiente em Sistema Holter, que não possui informação preliminar quanto ao arrolamento ou não dos sujeitos na pesquisa. Tanto os laudos quanto os formulários foram arquivados na forma impressa, para posterior análise de dados e processamento estatístico.

Este trabalho foi recebido para análise no Comitê de Ética e Pesquisa (CEP) da Universidade Tiradentes - UNIT em 29/11/2018, com o CAAE de número 85899118.7.0000.5371. Não houve conflito de interesse neste estudo. O estudo cumpriu os requisitos da resolução Conselho Nacional de Saúde 196/96 do CONEP e as determinações internacionais especificadas na Declaração de Helsinki para a realização de pesquisas em seres humanos. A estratégia de arrolamento foi espontânea, ou seja, decorreu de pacientes que procuraram o Serviço de Eletrocardiografia Ambulatorial em virtude de sua preferência, portanto não houve influência de membros do setor, direta ou indireta, no recrutamento de participantes ou na busca ativa de voluntários.

A análise estatística foi realizada de tal forma que as variáveis categóricas foram apresentadas em número absoluto e porcentagem enquanto as variáveis numéricas foram apresentadas em média e desvio-padrão. Além disso, o teste do quiquadrado de Pearson foi utilizado para medidas de associação entre variáveis categóricas e as variáveis numéricas foram apresentadas em média, SD, mediana e desvio-padrão. Depois disso, o padrão de normalidade de distribuição foi avaliado pelo teste de Shapiro-Francia e as comparações entre variáveis com padrão normal de distribuição foram estimadas mediante o teste t de Student.

Os resultados foram apresentados em estimativas pontuais (coeficientes) e medidas de dispersão foram calculadas mediante a obtenção de intervalos de confiança a 95\%. Considerou-se o valor de p bicaudal $<0,05$ como indicativo de significância estatística. Os cálculos e gráficos foram realizados em Stata 15.1.

\section{Resultados}

No grupo foram analisados 3988 pacientes dos quais 3730 se encontravam em ritmo sinusal (grupo controle) e possuíam uma idade média de 61,26 anos, com mínima de 40 anos e máxima de 98 anos. Deste grupo 2410 pacientes são do sexo feminino $(60,43 \%)$ e 1578 são do sexo masculino (39,57\%). Porém, dentre os pacientes, que possuíam ritmo de FA, foram observados e analisados em um estudo ancilar, uma amostra de 258 portadores de fibrilação atrial na monitorização eletrocardiográfica de $24 \mathrm{~h}$, que também haviam realizado ecocardiograma transtorácico. Neste grupo, foi observada uma idade média de 70,03 anos, com idade mínima de 40 anos e máxima de 97 anos, com desvio padrão de 11,66 anos. Destes, 166 $(64,34 \%)$ são do sexo feminino e $92(35,66 \%)$ são do sexo masculino.

Dentre demais resultados, na população estudada, 76 pacientes $(29,92 \%)$ possuíam um sistema cardiovascular sem demais alterações que não a FA, 137 (53,94\%) mostraram-se com alterações mínimas, 27 (10,63\%) foram observadas 
alterações moderadas e em 14 pacientes $(5,51 \%)$ foram vistas alterações graves, avaliadas no laudo eletrocardiográfico. Dentre os pacientes em ritmo sinusal foram vistos os achados: normais em 2059 pacientes (55,20\%), achados mínimos em 1489 $(39,84 \%)$, alterações moderadas em $150(4,05 \%)$ e alterações graves em $35(0,94 \%)$. Estes achados estão descritos na tabela 1 e ilustrados comparativamente nas Figuras 1 e 2.

Tabela 1 - Perfil da amostra em gênero, idade e alterações cardíacas.

Fibrilação Atrial Ritmo sinusal Valor de $\mathbf{p}$

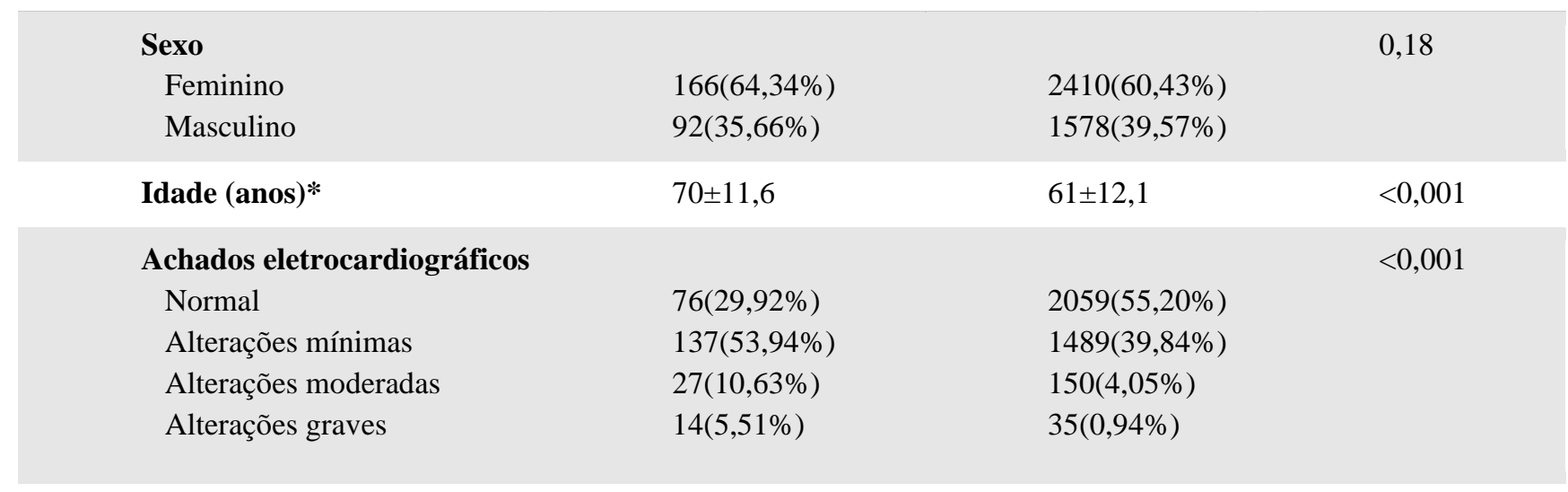

*: Média e desvio padrão. Evidencia-se o padrão de distribuição da população estudada de acordo com o sexo, idade, ritmo eletrocardiográfico e padrão de alterações encontradas que não FA. Fonte: Autores (2021).

Nesta tabela observa-se uma maior distribuição de achados mínimos moderados e graves nos pacientes em FA ilustrado em figuras a seguir, bem como uma maior idade, sendo ambas as populações predominantemente femininas.

Figura 1 - Prevalência de alterações cardíacas em indivíduos com FA.

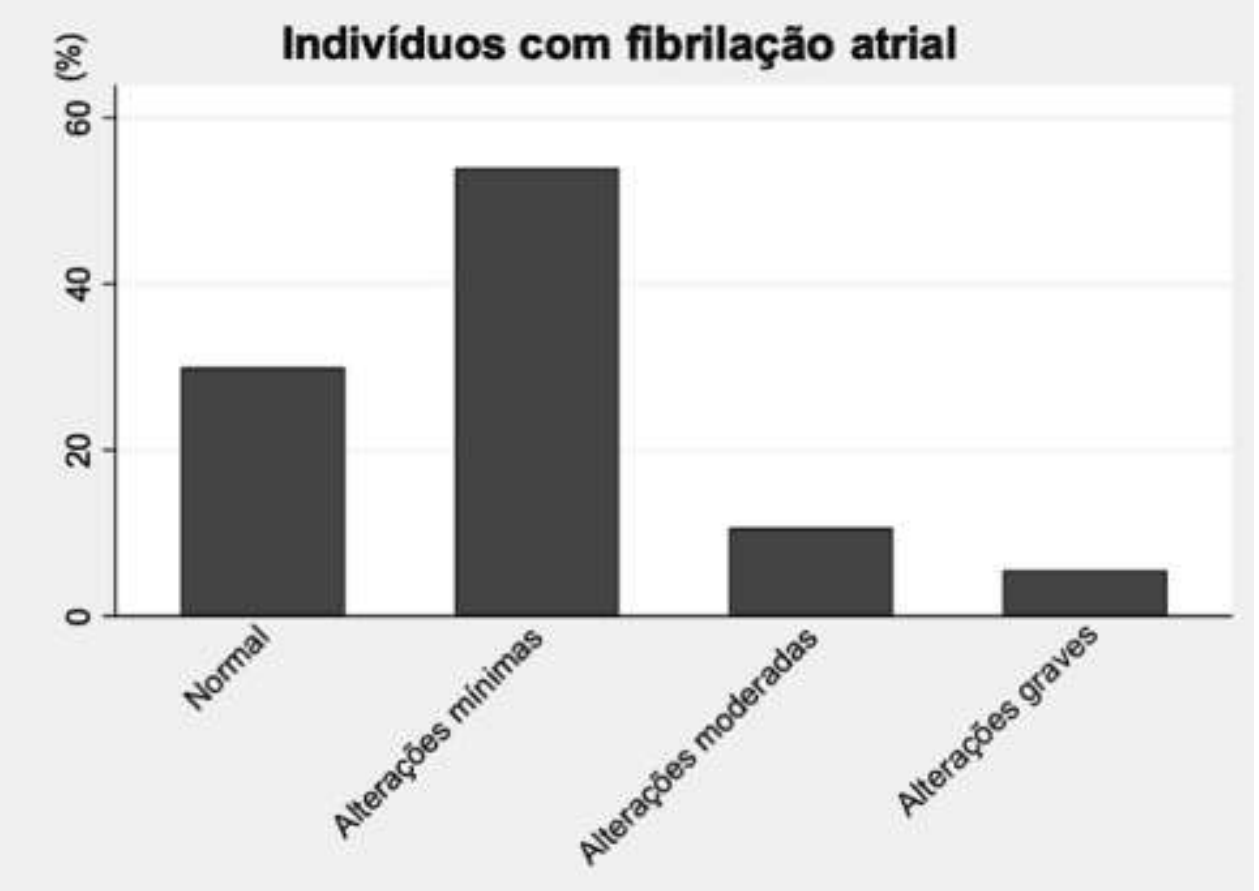

No gráfico é ilustrada a distribuição de alterações cardíacas que não FA nos pacientes portadores de FA. Fonte: Autores (2021).

No gráfico vê-se a ilustração da distribuição entre populações sem achados que não FA, alterações mínimas, moderadas e graves. 
Figura 2 - Prevalência de alterações cardíacas em indivíduos sem FA.

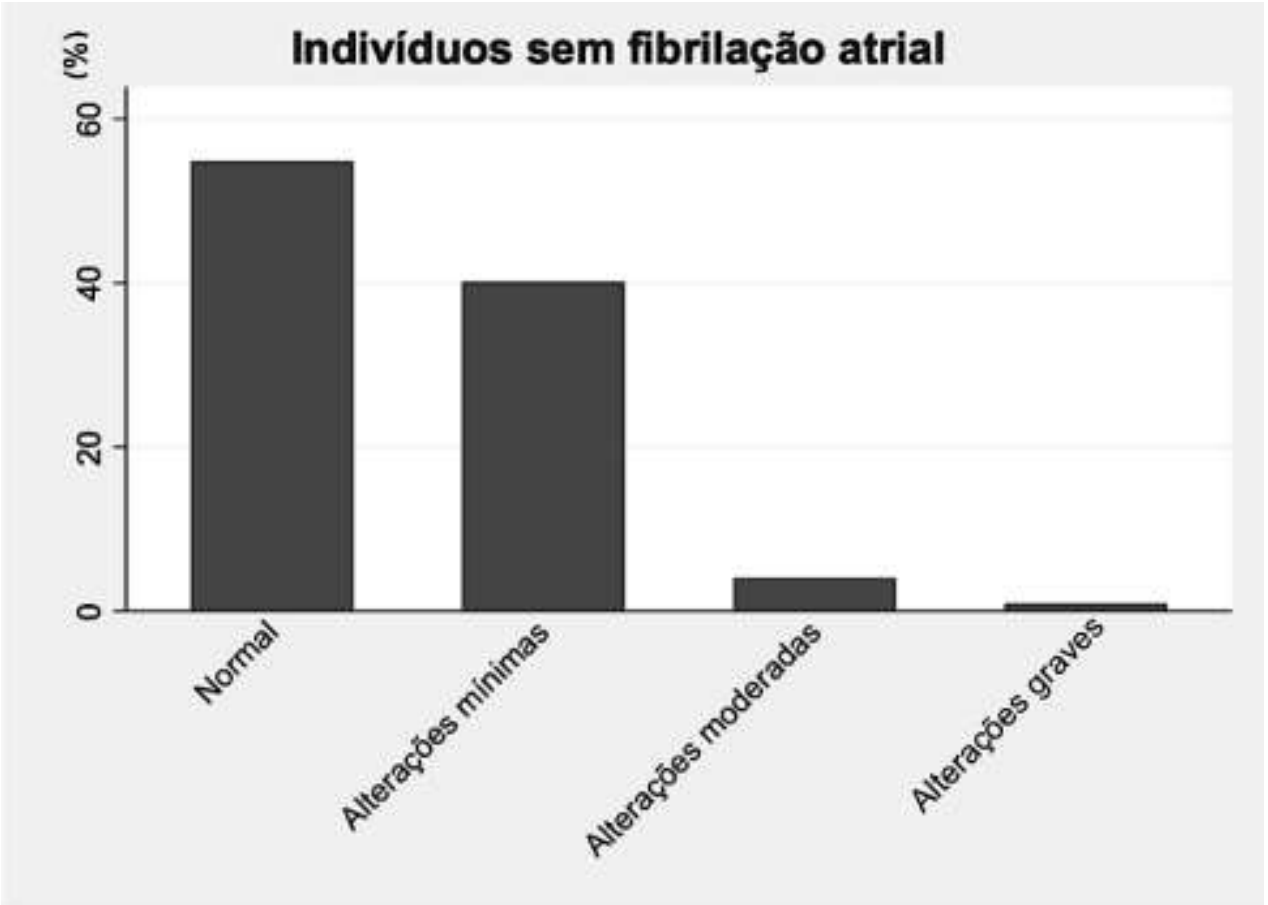

No gráfico é ilustrada a distribuição de alterações cardíacas que não FA nos pacientes portadores em ritmo sinusal. Fonte: Autores (2021).

Ao gráfico referido acima observa-se uma população em ritmo sinusal com padrao de distribuição bastante diferente daqueles em FA com maiores números de indivíduos sem alterações e alterações mínimas, bem como um número de alterações moderadas e graves significativamente menores.

Nos pacientes com achados de FA, a medida do diâmetro do AE teve uma média de 32,65 milímetros $( \pm 7,93)$, com um tamanho mínimo de $23 \mathrm{~mm}$ e máximo de $65 \mathrm{~mm}$, tal medida teve um valor de $\mathrm{p}<0.001$. Associada a essa informação, foi analisado o volume do AE na amostra para os quais foram obtidos resultados de uma medida média de $38,82 \mathrm{ml} / \mathrm{m}^{2}( \pm 17,95)$, tendo um valor máximo de $92,27 \mathrm{ml} / \mathrm{m}^{2}$ e um valor de $\mathrm{p}<0,001$, indicando um alto nível de confiança. Outra variável analisada foi a Fração de ejeção do ventrículo esquerdo que obteve uma média de 66,42\% ( $\pm 7,95)$, com mínima de 32\% e máxima de $85 \%$ e um valor de $\mathrm{p}<0,001$. Ainda, outra variável analisada em 258 participantes foi o diâmetro da aorta e nesta foi encontrado um valor médio de 32,65 mm $( \pm 3,97)$, valor mínimo de $19 \mathrm{~mm}$ e máximo de $42 \mathrm{~mm}$.

Além disso, algumas medidas foram observadas em um grupo reduzido de participantes, com variáveis analisadas na eletrocardiografia ambulatorial de 24h. A onda E, onda A, onda E', razão E:A e razão E:E' quando comparadas com as mesmas medidas no grupo em ritmo sinusal, se encontram na Tabela 3. Por fim, algumas medidas específicas foram ilustradas de modo comparativo entre os grupos na forma de gráficos em boxplot. Na figura 3 vê-se a medida do diâmetro da aorta nos grupos de portadores de FA e nos pacientes em ritmo sinusal, nesta medida não foram observadas alterações significativas além de uma ligeira elevação na mediana dos achados nos pacientes com FA. Na figura 3, observa-se o padrão de distribuição do diâmetro de átrio esquerdo entre pacientes com ritmo sinusal e fibrilação atrial sendo sua mediana ligeiramente superior em pacientes com FA, e maior número de valores extremos entre pacientes com ritmo sinusal. Em adição, na Figura 4 está ilustrado a diferença nas medidas do IVAE entre pacientes portadores de FA e em ritmo sinusal, nesta observa-se uma elevação significativa da mediana, percentis 25 e 75 além dos valores extremos superiores no grupo portador de FA.

Tais achados indicam a importância desta medida na avaliação cardiológica. Por fim, na figura 5 está a comparação na razão E:E' entre os grupos e está evidenciado uma elevação no percentil 25, mediana, percentil 74 e valores extremos superiores no grupo dos portadores de FA, indicando a importância deste achado para o objetivo da pesquisa. 
Tabela 2 - Parâmetros analisados.

\begin{tabular}{|lccc|} 
& Fibrilação Atrial** & Ritmo Sinusal ${ }^{* *}$ & Valor de p \\
\hline Diâmetro do AE $(\mathbf{m m})$ & $39,54( \pm 7,93)$ & $36,49( \pm 5,13)$ & $<0,001$ \\
\hline Volume do AE $(\mathbf{m l} / \mathbf{m} 2)$ & $38,82( \pm 17,95)$ & $26,05( \pm 9,37)$ & $<0,001$ \\
\hline Fração de ejeção $(\boldsymbol{\%}) *$ & $66,42( \pm 7,95)$ & $68,14( \pm 7,46)$ & $<0,001$ \\
\hline Diâmetro da aorta $(\mathbf{m m})$ & $32,65( \pm 3,97)$ & $32,04( \pm 4,17)$ & 0,02 \\
\hline Diâmetro diastólico $(\mathbf{m m}) *$ & $50,45( \pm 5,88)$ & $49,69( \pm 5,13)$ & 0,001 \\
\hline Diâmetro sistólico $(\mathbf{m m}) *$ & $31,54( \pm 5,88)$ & $30,52( \pm 5)$ & 0,05 \\
\hline Diâmetro septal $(\mathbf{m m})$ & $8,44( \pm 1,5)$ & $8,24( \pm 1,65)$ & \\
\hline
\end{tabular}

*: Do ventrículo esquerdo. **: Média e desvio padrão. Parâmetros ecocardiográficos analisados na população estudada mostrando-se em comparação entre aqueles em FA e ritmo sinusal. Fonte: Autores (2021).

Observa-se principalmente na tabela apresentada maiores medidas em Diâmetro do AE e Volume do AE naqueles com FA quando comparados com os pacientes em ritmo sinusal.

Tabela 3 - Medidas e razões eletrocardiográficas para avaliação da função diastólica.

\begin{tabular}{|llll} 
& Fibrilação Atrial* & Ritmo Sinusal* & Valor de p \\
\hline Onda e $(\mathbf{m m})$ & $71,4( \pm 19,1)$ & $70,45( \pm 18,43)$ & 0,67 \\
\hline Onda a $(\mathbf{m m})$ & $85,71( \pm 11,03)$ & $77,89( \pm 19,09)$ & 0,31 \\
\hline Onda e' $(\mathbf{m m})$ & $7,91( \pm 2,93)$ & $8,6( \pm 4,76)$ & 0,24 \\
\hline Razão e:a & $0,97( \pm 0,38)$ & $1,36( \pm 4,7)$ & 0,83 \\
\hline Razão e:e' & $10,51( \pm 3,99)$ & $9,06( \pm 3,05)$ & $<0,001$ \\
\hline
\end{tabular}

*: Média e desvio padrão. Parâmetros eletrocardiográficos analisados na população estudada mostrando-se em comparação entre aqueles em FA e ritmo sinusal. Fonte: Autores (2021).

Importante salientar na tabela acima o achado de razão E:E’ superior naqueles com FA, indicando uma propensão ma maior disfunção diastólica neste grupo. 
Figura 3 - Boxplot - Diâmetro do átrio esquerdo (mm).

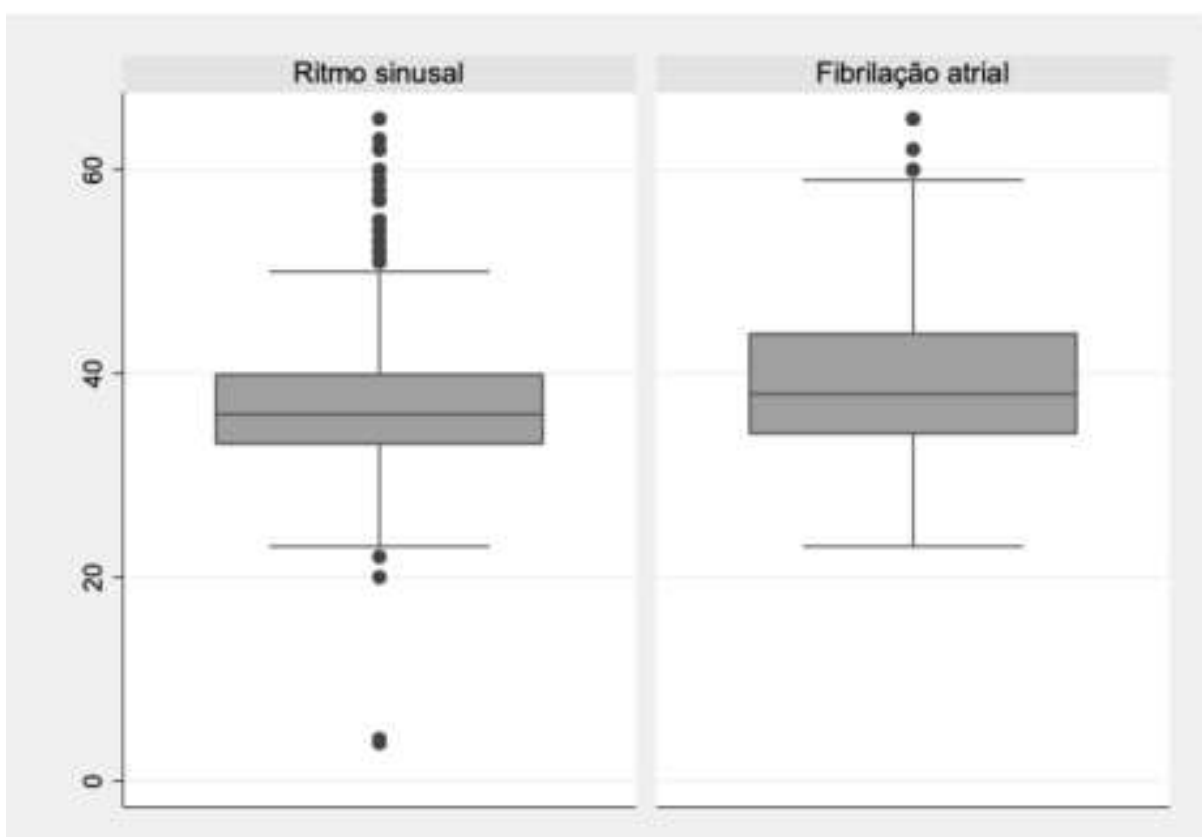

Padrão de distribuição comparativa de análise das medidas do diâmetro do átrio esquerdo entre os pacientes em FA e em ritmo sinusal. Fonte: Autores (2021).

Neste gráfico observa-se uma alteração no nível da mediana, representada pela linha no centro do retângulo cinza em amas as imagens, determinando uma maior alteração nesta medida naqueles com FA. Além disso, é de importância o percentil 75, representado pela base superior do retângulo, mostrando uma maior tendência do grupo de FA para alterações nesta medida para sua elevação quando comparado ao grupo em ritmo sinusal. Por fim, observa-se maiores valores extremos, ilustrado pelas linhas superiores no gráfico provido, corroborando com a maior tendência superior desta medida no grupo FA.

Figura 4 - Boxplot - Volume do átrio esquerdo (m1/m2).

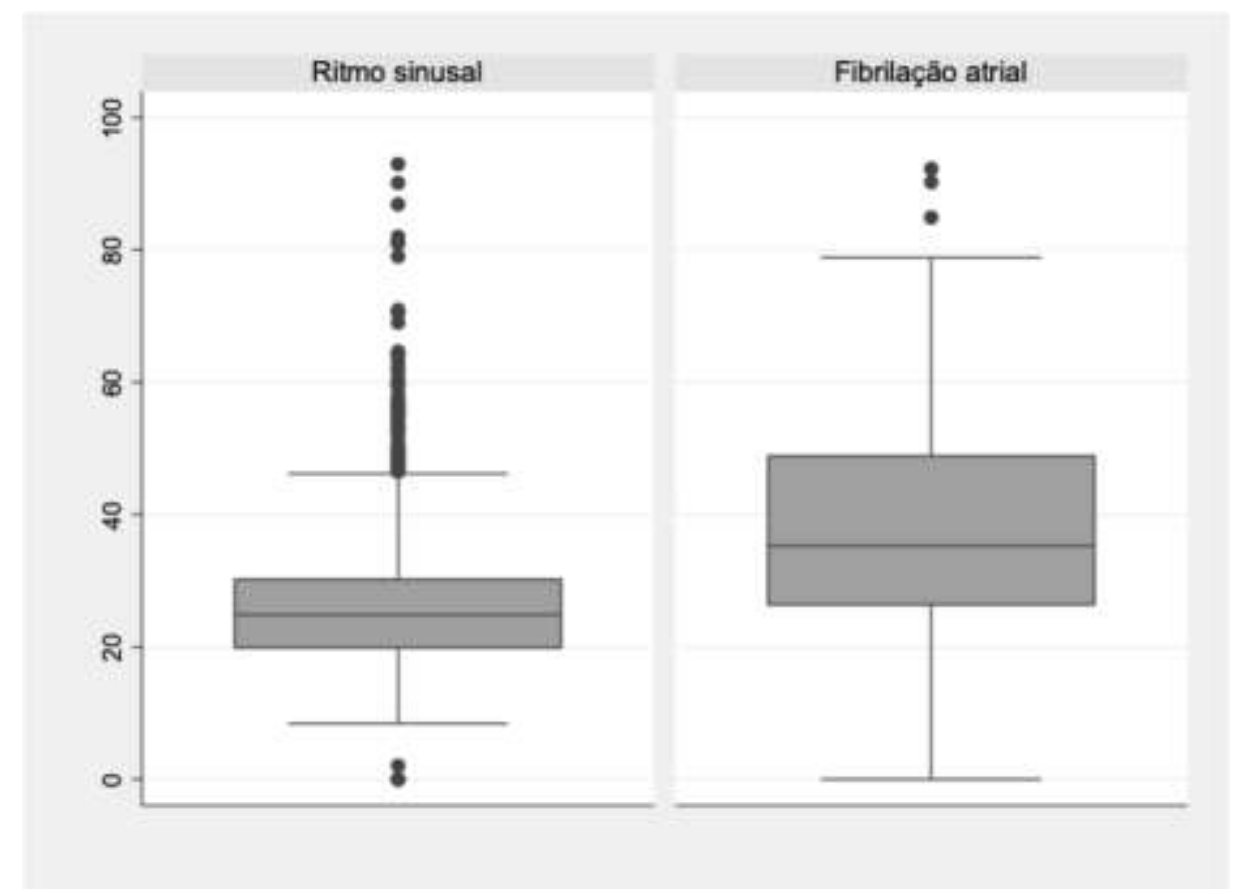

Padrão de distribuição comparativa de análise das medidas do volume do átrio esquerdo entre os pacientes em FA e em ritmo sinusal. Fonte: Autores (2021). 
Na ilustração vê-se uma maior alteração significativa nas medidas de percentil 25, representada pela base inferior do retângulo, mediana, percentil 75 e limite superior. Tais achados demonstram uma importante alteração na medida analisada naqueles pacientes portadores de FA.

Figura 5 - Boxplot - Razão E:E’.

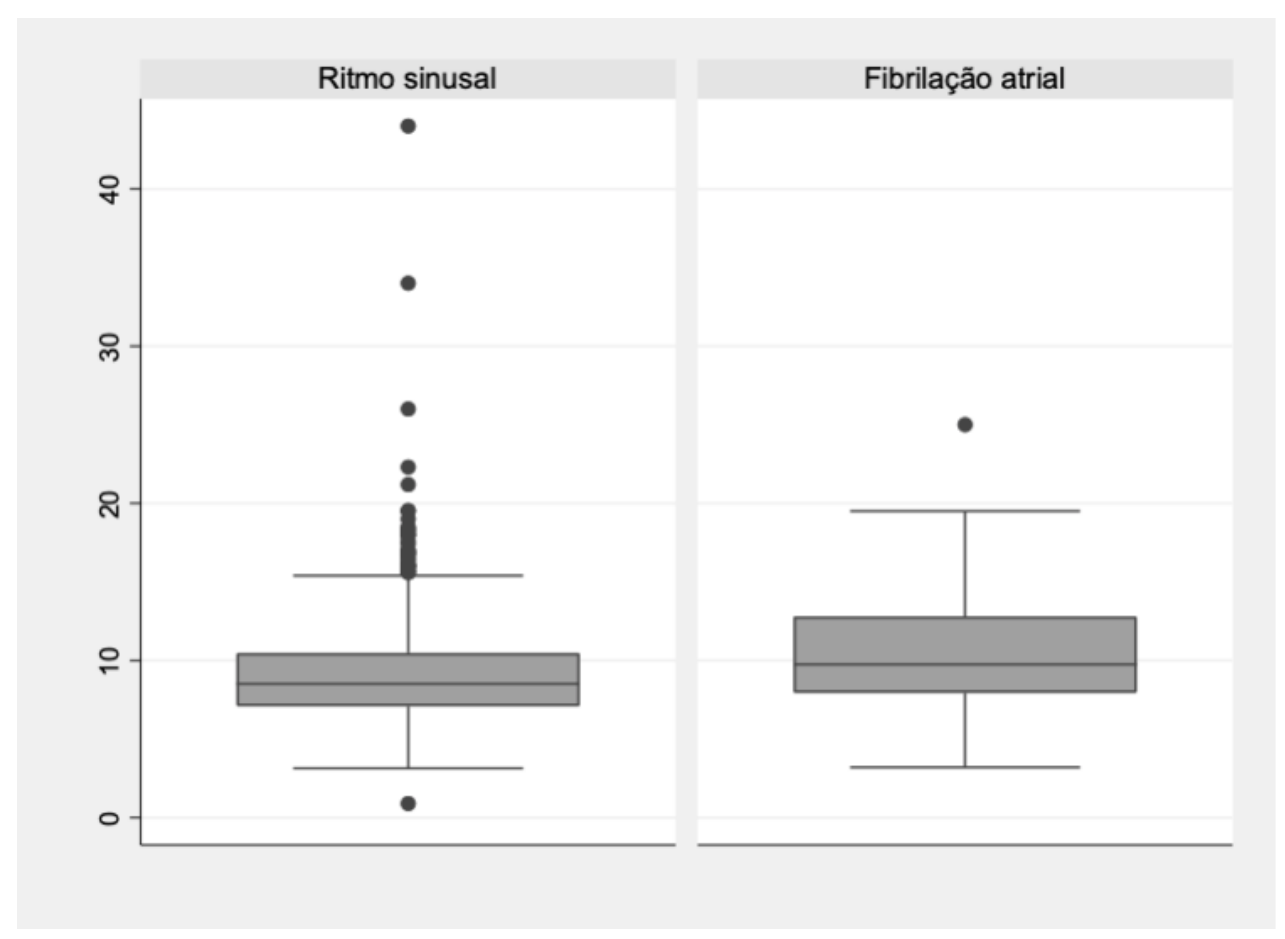

Padrão de distribuição comparativa de análise da razão E:E’entre os pacientes em FA e em ritmo sinusal. Fonte: Autores (2021).

Ainda, nesta análise observa-se uma elevação na mediana, no percentil 75 e valores superiores da análise naqueles em FA quando comparados aos em ritmo sinusal.

\section{Discussão}

Os achados encontrados corroboram em sua maioria com o perfil já relatado na literatura. Inicialmente o parâmetro com alteração mais significativa, e maior participação na fisiopatologia, foi o índice de volume do átrio esquerdo (IVAE), este é um achado sensível a alterações subagudas e crônicas, como na FA e que varia de acordo com o sexo e massa corporal do indivíduo analisado. Segundo a literatura, sua medida de normalidade é de $22( \pm 6) \mathrm{ml} / \mathrm{m} 2$ e com um limite superior de $34 \mathrm{ml} / \mathrm{m} 2$ (Secundo, et al., 2014; Kim \& Youn, 2011; Lang, et al., 2015).

No atual estudo observou-se um IVAE médio de 38,82( $\pm 17,95) \mathrm{ml} / \mathrm{m} 2$ nos pacientes com FA enquanto naqueles em ritmo sinusal a medida encontrada foi de $26,05( \pm 9,37)$ o que corrobora com a importância fisiopatológica para o desenvolvimento da patologia e confirma o IVAE como o achado de maior importância no ETT dos pacientes com FA, pois a alteração deste valor está intrinsecamente correlacionada com as alterações de substrato observadas principalmente no AE, além de poder ser utilizada como fator prognóstico, indicador de pior controle da FA, e menor índice de sucesso na terapia de cardioversão, indicando que esta medida pode ser estudada em foco para melhor esclarecimento no desencadeamento até o acompanhamento da FA (Kim \& Youn, 2011). Portanto, tais diferenças estão evidenciadas na figura 4.

Em acompanhamento a esta medida, outro achado de importância foi o diâmetro do AE, que segundo a literatura é uma medida importante para o desenvolvimento da FA, principalmente quando em acompanhamento de alterações na fração de ejeção (Zeng, et al., 2017). Ainda, o valor limítrofe da normalidade deste achado é de até $40 \mathrm{~mm}$, havendo somente um 
achado significativo no percentil 75 dos pacientes com FA, a qual tinha medida de $44 \mathrm{~mm}$, indicando que os pacientes portadores de FA possuem uma maior chance de possuir uma medida acima das referências de normalidade. Porém, por esta ser uma medida unidimensional de um órgão tridimensional esta possui uma sensibilidade reduzida e indica-se que a a superioridade da medida do IVAE para avaliação do aparecimento da FA. Ainda, esta medida pode ser utilizada como um preditor do IVAE, a qual possui maior sensibilidade e especificidade (Zeng, et al., 2017; Schvartzman, et al., 2000; Mills, et al., 2020). A medida do diâmetro do AE está ilustrada na figura 3.

Dentre os outros achados, não se observou uma diferença significativa nas medidas entre a população portadora de FA e em ritmo sinusal. Apesar disso, na literatura diversos parâmetros são importantes para a determinação do impacto da FA no funcionamento, analisados a partir do influxo entre sístoles, ou a função diastólica como a razão E:E', razão E:A, onda E e onda A, que foram analisadas neste estudo (Kotecha, et al., 2017; Sanchis, et al., 2018).

Além disso, outro achado de importância significativa foi a maior incidência de alterações diferentes da FA no exame analisado a partir do dos achados eletrocardiográficos, com maior prevalência de achados moderados a graves. Neste estudo foi observado uma redução significativa naqueles sem alterações que não a FA, 76\% se enquadravam na normalidade para os em ritmo sinusal e contraste a somente 29,92\% naqueles com FA. Em consequente, seguindo este parâmetro, os pacientes tiveram um índice maior de alterações mínimas, moderadas e graves, 53,94\%,10,63\% e 5,51\%, respectivamente, em comparação com os índices do grupo em ritmo sinusal de 40,16\%, 4,05\% e 0,94\%. Tais achados indicam que os pacientes com a fibrilação possuem um maior risco de desenvolvimento de patologias leves, moderadas e graves quando comparadas com a população em ritmo sinusal. Porém, tal comparação pode ser limitada pela diferença nas idades entre os grupos de cerca de nove anos entre as idades médias e pelo número reduzido dos portadores de FA quando comparado ao grupo de ritmo sinusal.

A partir das informações explanadas, o atual estudo possui algumas limitações. Os pacientes foram selecionados de forma unicêntrica, o registro de amostragem se deu de uma forma não probabilística, há uma variabilidade significativa no número da amostra dos pacientes com FA quando em comparação a aqueles em ritmo sinusal, por tratar de um estudo transversal, e pela não medição de estudos bioquímicos para afastar comorbidades que possam influenciar no resultado. Porém, trata-se de um estudo de valor devido ao grupo amostras significativas e por ser um estudo com variáveis diversas analisadas em um tema não explorado.

\section{Conclusão}

Em suma, o achado mais importante para os pacientes com FA foi o IVAE, o qual teve um resultado elevado no grupo portadores de FA, seguida pelo diâmetro do AE. Ainda, as demais variáveis analisadas não possuíram alterações significativas. Por fim, a partir da análise ecocardiográfica, foi observado que os pacientes com FA possuíam maior concomitância de alterações leves, moderadas e graves, se comparado com indivíduos em ritmo sinusal.

\section{Referências}

Crawford, M. H., Bernstein, S. J., Deedwania, P. C., DiMarco, J. P., Ferrick, K. J., Garson Jr, A., \& Smith Jr, S. C. (1999). ACC/AHA guidelines for ambulatory electrocardiography: executive summary and recommendations: a report of the American College of Cardiology/American Heart Association task force on practice guidelines (committee to revise the guidelines for ambulatory electrocardiography) developed in collaboration with the north American society for pacing and electrophysiology. Circulation, 100(8), 886-893.

DiMarco, J. P., \& Philbrick, J. T. (1990). Use of ambulatory electrocardiographic (Holter) monitoring. Annals of internal medicine, 113(1), 53-68.

Estrela, C. (2018). Metodologia científica: ciência, ensino, pesquisa. Artes Médicas.

Field, T. S., Weijs, B., Curcio, A., Giustozzi, M., Sudikas, S., Katholing, A., \& Martinez, C. (2019). Incident atrial fibrillation, dementia and the role of anticoagulation: a population-based cohort study. Thrombosis and haemostasis, 119(06), 981-991.

Hindricks, G., Potpara, T., Dagres, N., Arbelo, E., Bax, J. J., Blomström-Lundqvist, C., \& Watkins, C. L. (2021). 2020 ESC Guidelines for the diagnosis and management of atrial fibrillation developed in collaboration with the European Association for Cardio-Thoracic Surgery (EACTS) The Task Force for the 
diagnosis and management of atrial fibrillation of the European Society of Cardiology (ESC) Developed with the special contribution of the European Heart Rhythm Association (EHRA) of the ESC. European heart journal, 42(5), 373-498.

John, A. G., Hirsch, G. A., \& Stoddard, M. F. (2018). Frequent premature atrial contractions impair left atrial contractile function and promote adverse left atrial remodeling. Echocardiography, 35(9), 1310-1317.

Jonas, D. E., Kahwati, L. C., Yun, J. D., Middleton, J. C., Coker-Schwimmer, M., \& Asher, G. N. (2018). Screening for atrial fibrillation with electrocardiography: evidence report and systematic review for the US Preventive Services Task Force. Jama, 320(5), $485-498$.

Kennedy, H. L. (2006). The history, science, and innovation of Holter technology. Annals of Noninvasive Electrocardiology, 11(1), 85-94.

Kim, T. S., \& Youn, H. J. (2011). Role of echocardiography in atrial fibrillation. Journal of Cardiovascular Ultrasound, 19(2), 51-61.

Kirchhof, P., Benussi, S., Kotecha, D., Ahlsson, A., Atar, D., Casadei, B., \& Grupo de Trabajo de la Sociedad Europea de Cardiología. (2017). Guía ESC 2016 sobre el diagnóstico y tratamiento de la fibrilación auricular, desarrollada en colaboración con la EACTS. Revista Española de Cardiología, 70(1), 50-e1.

Kotecha, D., Mohamed, M., Shantsila, E., Popescu, B. A., \& Steeds, R. P. (2017). Is echocardiography valid and reproducible in patients with atrial fibrillation? A systematic review. Ep Europace, 19(9), 1427-1438.

Lang, R. M., Badano, L. P., Mor-Avi, V., Afilalo, J., Armstrong, A., Ernande, L., \& Voigt, J. U. (2015). Recommendations for cardiac chamber quantification by echocardiography in adults: an update from the American Society of Echocardiography and the European Association of Cardiovascular Imaging. European Heart Journal-Cardiovascular Imaging, 16(3), 233-271.

Mills, H., Espersen, K., Jurlander, R., Iversen, K., Bundgaard, H., \& Raja, A. A. (2020). Prevention of sudden cardiac death in hypertrophic cardiomyopathy: Risk assessment using left atrial diameter predicted from left atrial volume. Clinical cardiology, 43(6), 581-586.

Morganroth, J. (1985). Ambulatory Holter electrocardiography: choice of technologies and clinical uses. Annals of internal medicine, 102(1), 73-81.

Ricci, B., Chang, A. D., Hemendinger, M., Dakay, K., Cutting, S., Burton, T., ... \& Yaghi, S. (2018). A simple score that predicts paroxysmal atrial fibrillation on outpatient cardiac monitoring after embolic stroke of unknown source. Journal of Stroke and Cerebrovascular Diseases, $27(6)$, $1692-1696$.

Sanchis, L., Andrea, R., Falces, C., Poyatos, S., Vidal, B., \& Sitges, M. (2018). Differential clinical implications of current recommendations for the evaluation of left ventricular diastolic function by echocardiography. Journal of the American Society of Echocardiography, 31(11), 1203-1208.

Schvartzman, P. R., Fuchs, F. D., Mello, A. G., Coli, M., Schvartzman, M., \& Moreira, L. B. (2000). Valores normais de medidas ecocardiográficas. Um estudo de base populacional. Arq Bras Cardiol, 75(2), 107-10.

Secundo, J. A., Santos, M. A. A., Faro, G. B. D. A., Soares, C. B., Silva, A. M. P., Secundo, P. F. C., \& Sousa, A. C. S. (2014). Indice de volume atrial esquerdo e predição de eventos em síndrome coronária aguda: registro solar. Arq Bras Cardiol, 103, 2, 82-291.

Soeki, T., Matsuura, T., Tobiume, T., Bando, S., Matsumoto, K., Nagano, H., ... \& Sata, M. (2018). Clinical, electrocardiographic, and echocardiographic parameter combination predicts the onset of atrial fibrillation. Circulation Journal, 82(9), 2253-2258.

Zegkos, T., Efthimiadis, G. K., Parcharidou, D. G., Gossios, T. D., Giannakoulas, G., Ntelios, D., \& Karvounis, H. I. (2017). Atrial fibrillation in hypertrophic cardiomyopathy: A turning point towards increased morbidity and mortality. Hellenic Journal of Cardiology, 58(5), 331-339.

Zeng, R. X., Chen, M. S., Lian, B. T., Liao, P. D., \& Zhang, M. Z. (2017). Left ventricular ejection fraction and left atrium diameter related to new-onset atrial fibrillation following acute myocardial infarction: a systematic review and meta-analysis. Oncotarget, 8(46), 81137. 